Neuroimage. 2010 February 1; 49(3): 1991-2000. doi:10.1016/j.neuroimage.2009.08.052.

\title{
Object Familiarity Modulates Effective Connectivity During Haptic Shape Perception
}

\author{
Gopikrishna Deshpande ${ }^{1}$, Xiaoping Hu${ }^{1}$, Simon Lacey ${ }^{2}$, Randall Stilla ${ }^{2}$, and K. \\ Sathian $2,3,4,5$ \\ ${ }^{1}$ Coulter Department of Biomedical Engineering, Emory University \& Georgia Institute of \\ Technology, Atlanta, GA, USA \\ ${ }^{2}$ Department of Neurology, Emory University, Atlanta, GA, USA \\ ${ }^{3}$ Department of Rehabilitation Medicine, Emory University, Atlanta, GA, USA \\ ${ }^{4}$ Department of Psychology, Emory University, Atlanta, GA, USA \\ ${ }^{5}$ Rehabilitation R\&D Center of Excellence, Atlanta VAMC, Decatur, GA, USA
}

\begin{abstract}
In the preceding paper (Lacey et al., 2009), we showed that the activations evoked by visual imagery overlapped more extensively, and their magnitudes were more correlated, with those evoked during haptic shape perception of familiar, compared to unfamiliar, objects. Here we used task-specific analyses of functional and effective connectivity to provide convergent evidence. These analyses showed that the visual imagery and familiar haptic shape tasks activated similar networks, whereas the unfamiliar haptic shape task activated a different network. Multivariate Granger causality analyses of effective connectivity, in both a conventional form and one purged of zero-lag correlations, showed that the visual imagery and familiar haptic shape networks involved top-down paths from prefrontal cortex into the lateral occipital complex (LOC), whereas the unfamiliar haptic shape network was characterized by bottom-up, somatosensory inputs into the LOC. We conclude that shape representations in the LOC are flexibly accessible, either topdown or bottom-up, according to task demands, and that visual imagery is more involved in LOC activation during haptic shape perception when objects are familiar, compared to unfamiliar.
\end{abstract}

\section{Keywords}

Multisensory; cross-modal; visual cortex; lateral occipital complex; somatosensory cortex; fMRI; Granger causality analysis; visual imagery

\section{Introduction}

In the preceding paper (Lacey et al., 2009), we showed that the extent to which haptic shape perception (HS) shares neural circuitry with visual imagery (VI) depends on familiarity of the objects used during the haptic shape task: For unfamiliar, meaningless objects, the extent

Corresponding author: K. Sathian, Department of Neurology, Emory University School of Medicine, WMB-6000, 101 Woodruff Circle, Atlanta GA 30322, USA, Tel: 404-727-1366 Fax: 404-727-3157 krish.sathian@emory.edu.

Publisher's Disclaimer: This is a PDF file of an unedited manuscript that has been accepted for publication. As a service to our customers we are providing this early version of the manuscript. The manuscript will undergo copyediting, typesetting, and review of the resulting proof before it is published in its final citable form. Please note that during the production process errors may be discovered which could affect the content, and all legal disclaimers that apply to the journal pertain. 
of overlap of shape-selective activation with activation during visual shape imagery is limited, and most of the overlapping foci do not exhibit correlated activation magnitudes across subjects between the HS and VI tasks. In contrast, the activity evoked during HS perception using familiar objects overlaps much more extensively with that evoked during VI, and activation magnitudes across subjects are significantly correlated between the HS and VI tasks in a number of the overlap zones, including the lateral occipital complex (LOC) bilaterally. These findings suggest that recruitment of the LOC during HS perception reflects VI when the palpated objects are familiar, but not when they are unfamiliar.

In this paper, we present converging evidence on the role of VI during HS, using analysis of connectivity during task performance. Since VI involves top-down paths from prefrontal and posterior parietal cortex into visual cortex (Mechelli et al., 2004), imagery mediation of LOC recruitment would imply analogous top-down paths into the LOC during both the VI and HS tasks. An alternative hypothesis is that LOC recruitment during HS perception reflects engagement of a multisensory representation of shape via bottom-up projections from somatosensory cortex. This would be favored by the existence of bottom-up paths into the LOC from somatosensory cortical areas. Earlier studies of effective connectivity (EC) during HS were consistent with the idea that there are both bottom-up paths from somatosensory cortex, and top-down paths from postero-supero-medial parietal cortex, into the LOC (Peltier et al., 2007; Deshpande et al., 2008). These studies, however, were based on the use of unfamiliar objects only, and also did not analyze EC by task. Here we analyzed task-specific EC during VI, HS perception of unfamiliar objects and HS perception of familiar objects using data presented in the preceding paper to test specific predictions about paths into the LOC: that the LOC would be driven primarily top-down from prefrontal and parietal cortex during VI and HS perception of familiar objects, but by bottom-up paths from somatosensory cortex during HS perception of unfamiliar objects. Further, we tested the more general predictions that connectivity patterns during VI would be similar to those during HS for familiar, but not unfamiliar objects. A set of regions of interest (ROIs) was chosen to test these predictions, as elaborated later.

EC was analyzed using Granger causality (GC), which infers causality between two time series by means of cross-prediction: if future values of time series $y(t)$ can be predicted from past values of time series $x(t)$, then $x(t)$ can be inferred to have a causal influence on $y(t)$ (Granger, 1969). EC between a number of ROIs can be assessed by applying this approach, in a multivariate manner, to the time series of blood oxygenation level-dependent (BOLD) signal intensities from selected ROIs, as described in previous reports from our group (Stilla et al., 2007, 2008; Deshpande et al., 2008). Unlike earlier, bivariate, implementations of GC analysis which could only probe inputs to and outputs from one region at a time, the multivariate approach allows simultaneous assessment of all potential interactions between the selected ROIs while filtering out indirect effects mediated through intermediate nodes in the network. While our previous studies examined EC using the entire time series data, here we employed the same approach in a task-specific manner.

Recent work from our group (Deshpande et al., 2009) has examined the relation between $\mathrm{GC}$ and the commonly used functional connectivity (FC), which is based on instantaneous correlations between BOLD time series in various regions (Büchel and Friston, 2001). Previous studies have likened instantaneous GC, obtained from the vector autoregressive model (VAR) used to compute GC, to zero-lag correlation (Roebroeck et al, 2005). However, since instantaneous GC and time-lagged GC are derived from a common parameter - the variance of the VAR model error - the causality measures obtained are not independent of zero-lag correlation effects. Using simulations and analyses of functional magnetic resonance imaging (fMRI) data from a verbal working memory task, we showed that zero-lag correlations can leak into time-lagged GC estimates based on the hemodynamic 
response, owing to temporal blurring of neuronal activity (Deshpande et al., 2009). We therefore introduced a method to eliminate the effect of such zero-lag correlations from GCbased networks by explicitly modeling the zero-lag influences in the VAR model and then excluding their contribution to causal influences; we term this analysis correlation-purged GC (CPGC). Here we apply this method to the task-specific GC analysis of VI and HS outlined above. The resulting CPGC-based network can be considered to conservatively estimate directional interactions (i.e., EC) as inferred from BOLD time series, while the original GC-based network includes the true EC as well as the FC that leaked into the causal domain and thus spuriously appeared as EC. We present the results of both GC and CPGC analyses, and also report networks based on zero-lag correlation, corresponding to the commonly computed FC. It should be noted that zero-lag correlation-based (FC-based) networks in the present work as well as previous studies may include not only true instantaneous neuronal correlations, but also, potentially, directional neuronal interactions that occur too fast to be captured by the sluggish BOLD response. Examining all three types of analysis affords a complete picture of the network interactions, as reflected in the hemodynamic response.

\section{Materials and Methods}

Details regarding participants, tasks, image acquisition and image analysis are provided in the preceding paper (Lacey et al., 2009). Briefly, we conducted two experiments, with eight neurologically normal participants in each experiment (one person participated in both experiments). In each experiment, there was a session comprising runs of a VI task that required discrimination of the shape of visually imaged objects in response to hearing the object names, with a control condition involving discrimination of words from non-words (WnW task). Each experiment also included a separate session comprising runs of a condition that required HS discrimination, the control for this being a condition requiring haptic texture (HT) discrimination. In Experiment 1, the HS task used unfamiliar, meaningless objects whereas in Experiment 2, the HS task used familiar objects. In other respects, the two experiments were identical. In each experiment, activations in the VI and HS condition were first identified relative to their respective control conditions, after which conjunction analyses were used to reveal voxels active in both VI and HS conditions.

Connectivity analyses were performed on selected regions of interest (ROIs) constrained to be no larger than $125 \mathrm{~mm}^{3}(5 \times 5 \times 5 \mathrm{~mm}$ cube), and centered on centers of gravity of particular activations or overlap zones. A set of eighteen ROIs was chosen to test our a priori hypotheses. We began with the overlap zones identified by the conjunction of voxels active on both the VI task and the HS task (relative to their respective control conditions). There were 11 such overlap zones from Experiment 2, i.e., zones of overlap between activations evoked during VI and those evoked during familiar HS. These included the LOC foci which were also overlap zones in Experiment 1, i.e., zones of overlap between activations evoked during VI and those evoked during unfamiliar HS. Further, we also included the two additional overlap zones that were found only in Experiment 1 but not Experiment 2. Thus, altogether, there were 13 overlap zones that were present in at least one of the two experiments (Table 3 of Lacey et al., 2009). Apart from the LOCs, this set of ROIs included a number of prefrontal and parietal cortical foci that could be sources of top-down drive into the LOCs.

Next, we added four ROIs: bilateral postcentral sulcus (PCS), left anterior intraparietal sulcus (aIPS) and left posterior intraparietal sulcus (pIPS), that were common to both HS tasks (Table 8 of Lacey et al., 2009). The PCS corresponds to Brodmann's area 2 (Grefkes et al., 2001), which is part of primary somatosensory cortex, and could be the source of bottom-up somatosensory inputs into the LOC; while the two IPS areas could potentially 
funnel either bottom-up or top-down inputs into the LOC. Finally, the left frontal eye field (FEF) was included as a region active during VI and both HS tasks, although there was no significant overlap between activations due to VI and those due to HS (Tables 1, 2, 4, 5 of Lacey et al., 2009). Non-human primate studies have shown that ventral visual areas such as V4 are modulated by FEF activity during visual tasks (Moore and Armstrong, 2003); inclusion of the FEF allowed us to test the possibility that LOC activity during haptic perception is influenced by the FEF. Note that the LOC and PCS were the only paired ROIs, all other ROIs being left-sided. In what follows, therefore, the side of the ROI will be mentioned only for the LOC and the PCS.

The task-specific time series for these ROIs were extracted from the respective task blocks. These data were averaged across voxels within each ROI, normalized across runs and subjects, and concatenated across all runs and subjects to form a single vector per ROI. Using methods described previously (Stilla et al., 2007; Deshpande et al., 2008), a multivariate VAR model of the time series data was used to compute a directed transfer function for each potential interaction among the selected ROIs, thus generating a GC-based connectivity matrix for each task. As detailed elsewhere (Deshpande et al., 2009) and summarized below, we also computed the FC network and the CPGC-based network for each task by explicitly modeling the zero-lag term in the autoregressive model and using this term to derive the FC-based networks, but omitting this term in computing the CPGC-based networks.

Given $n$ time series $X(t)=\left[x_{1}(t) x_{2}(t) \ldots x_{n}(t)\right]$, the traditional VAR model of order $p$ is given by:

$$
X(t)=A(1) X(t-1)+A(2) X(t-2)+\cdots+A(p) X(t-p)+E(t)
$$

where $A(1) \ldots A(p)$ are the coefficients of the model and $E(t)$ is the model error. We previously reported a multivariate GC measure derived based on the model coefficients $A(1)$ $\ldots A(p)$ (Stilla et al., 2007, 2008; Deshpande et al., 2008). In order to account for the zerolag correlation effects, we introduced the zero-lag term into Eq.1. Thus,

$X(t)=A^{\prime}(0) X(t)+A^{\prime}(1) X(t-1)+A^{\prime}(2) X(t-2)+\cdots+A^{\prime}(p) X(t-p)+E^{\prime}(t)$

where the diagonal elements of $\mathrm{A}^{\prime}(0)$ are zero such that only the instantaneous crosscorrelation, and not the auto-correlation, between the time series are modeled. The inclusion of the zero-lag term affects the value of other coefficients and hence $A^{\prime}(1) \ldots A^{\prime}(p) \neq A(1)$ $\ldots A(p)$. Furthermore, GC obtained from $A^{\prime}(1) \ldots A^{\prime}(p)$ are cleansed of zero-lag correlation; we term the result correlation-purged GC (CPGC). In addition, the value of $A^{\prime}(0)$ represents the zero-lag correlation between the time series. It is to be noted that temporal dispersion caused by hemodynamic blurring could cause interactions between zero-lag and time-lagged correlations. CPGC estimated from the above procedure is likely to make it free from the "leakage" of zero-lag correlation into the causal domain under the assumption of linearity.

For the GC and CPGC analyses, model order was taken as 1, i.e., Granger causality was computed using the minimum time lag of one repetition time (TR, $2 \mathrm{~s}$ ). The statistical significance of the path weights in the task-specific connectivity matrices was determined using surrogate data, as detailed previously (Stilla et al., 2007; Deshpande et al., 2008). To assess the similarity between the networks (of each type) underlying each task, 2D correlation coefficients were computed between the task-specific connectivity matrices for 
the task-pairs in each experiment (VI and HS) and also between those for familiar and unfamiliar HS, as well as for the VI task in the two experiments: it should be noted that these latter two task-pairs used data from different experiments, with subject groups that were different with the exception of a single common subject. Further, we computed intertask correlations between VI and HS in each experiment for the weights of significant paths that were common to both tasks.

\section{Results}

Figures 1 and 2 illustrate the significant connections, and their directions, that emerged from multivariate connectivity analyses for the task-pairs in Experiments 1 and 2, respectively, in the conventional GC analyses (left panels), FC analyses (middle panels) and CPGC analyses (right panels). Tables 1-12 show the path weights for all interactions in the connectivity matrices for each condition in each type of analysis, with the significant paths shown in bold type. All significant path weights in the conventional GC analyses as well as the CPGC analyses co-varied, i.e., the BOLD signal for the two ROIs connected by each of these paths tended to rise or fall together, albeit with a phase difference (Stilla et al., 2007; Deshpande et al., 2008). While this type of co-variation is analogous to a positive correlation, this does not necessarily imply that the paths are excitatory, because the imaging data and connectivity analyses are based on the hemodynamic response, whose relationship with synaptic excitation and inhibition is still unclear. The three types of analysis are considered in turn below.

\section{GC analyses}

VI-During VI, the connectivity matrices were very similar in both experiments (compare top left panels in Figures 1 and 2, and path weights in Tables 1 and 2). In both experiments, the LOCs were driven largely from prefrontal ROIs, with significant inputs to both LOCs from the inferior frontal gyrus (IFG) and the orbitofrontal cortex (OFC). This fits with the expected pattern of top-down drive into the LOCs. Further, these regions (the LOCs, IFG and $\mathrm{OFC}$ ) were reciprocally connected, and the largest number of paths overall emanated from the IFG. Another set of reciprocal connections involved the dorsal premotor cortex (PMd), ventral premotor cortex (PMv) and the pulvinar region of the thalamus (Pul).

Unfamiliar HS perception-During unfamiliar HS (Figure 1, bottom left panel; Table 3), unlike during VI, the most prominent overall source was the right PCS, which was also the only ROI to drive both LOCs, while both right and left PCS drove the right LOC. These paths signify the presence of bottom-up inputs into the LOC, which were all reciprocated. There were also reciprocal interactions between the left LOC and anterior-ventral IPS (avIPS), and a path from the medial superior frontal gyrus (mSFG) to the left LOC. The latter path may reflect some top-down drive into the LOC. Unlike VI (see above) or familiar HS (see below), the PCS provided significant inputs to regions of the IPS: the left PCS drove the anterior IPS (aIPS) and superomedial IPS (smIPS) foci, and the right PCS drove the avIPS and smIPS foci. All but the last of these paths were reciprocal. The FEF was reciprocally connected with the aIPS; it also drove the smIPS and received input from the $\mathrm{mSFG}$ and PMd foci.

Familiar HS perception-Finally, during familiar HS (Figure 2, bottom left panel; Table 4), the IFG was a major source of outputs, as was the mSFG. The IFG drove both LOCs while the OFC drove the left LOC, once again consistent with top-down inputs into the LOCs, which were reciprocated - this pattern was similar to that found for VI. Strong reciprocal connections were found between the mid-fusiform (MF) focus and both LOCs. The posterior-ventral IPS (pvIPS) drove both LOCs with reciprocal drive from the left LOC, 
and there was another set of reciprocal connections involving the IFG, PMd and Pul region. The FEF was reciprocally connected with the PCS and avIPS.

Comparisons between tasks-Comparing these GC connectivity patterns between tasks suggests that the VI task was associated with similar connectivity patterns as the familiar HS task, but not the unfamiliar HS task. Consistent with this impression, the 2D correlation between connectivity matrices was highly significant for VI and familiar HS ( $\mathrm{r}=$ $0.51, \mathrm{p}<10^{-10}$, data from Expt. 2) but insignificant between VI and unfamiliar HS ( $\mathrm{r}=$ $-0.01, \mathrm{p}=0.91$, data from Expt. 1$)$. There was, interestingly, no significant correlation $(\mathrm{r}=$ $0.04, p=0.51$ ) between the two HS conditions, although these nominally involved the same task (the two HS conditions differed only with respect to object familiarity). This was not simply due to different subject groups for the two tasks, since the 2D correlations between the two experiments for the VI task, which also involved different subject groups, was very high $\left(\mathrm{r}=0.97, \mathrm{p}<10^{-15}\right)$.

\section{FC analyses}

VI—During VI (middle panels, top rows, Figures 1 and 2; Tables 5 and 6), the FC analyses revealed two essentially segregated networks - zero-lag correlations were present between areas within each of these networks but not between areas belonging to the different networks. In both experiments, one of these networks involved the LOCs, the pulvinar and a number of prefrontal regions: OFC, IFG, PMv, and PMd. The other network in Experiment 1 comprised the left PCS, and multiple parts of the IPS (aIPS, smIPS, pIPS and avIPS); in Experiment 2, this network included all these regions and also the right PCS, the FEF and the posterior fusiform gyrus (PF). Consistent with the GC analysis of VI, this FC analysis suggested that the LOCs interacted primarily with prefrontal cortical regions, including the two (OFC, IFG) that were the source of drive to the LOCs in the GC analysis.

HS perception-During unfamiliar HS (Figure 1, bottom row, middle panel; Table 7), the interactions were much more numerous than during VI, and there was no clear pattern of segregation into networks. It is notable that the IPS foci played a prominent part in the interactions, along with the PCS, LOC, FEF, PMv and OFC. During familiar HS (Figure 2, bottom row, middle panel; Table 8), the FC analysis revealed networks that were very similar to those in VI, with the same basic pattern of segregation. The network featuring the LOCs included all the regions found during VI (pulvinar, OFC, IFG, PMv, and PMd) and also the MF. That featuring the PCS regions included the same regions as during VI in Experiment 2. Many of the same paths were significant both during VI and familiar HS, although there were some differences. Whereas the two networks were strictly segregated during VI, during familiar HS there was a single path, from FEF to PMv, that violated this boundary.

Comparisons between tasks-The $2 \mathrm{D}$ correlation between connectivity matrices was again highest for the VI - familiar HS pair (data from Experiment 2; $\mathrm{r}=0.82, \mathrm{p}=5 \times 10^{-7}$ ), and also quite high for the VI conditions in the two experiments $\left(\mathrm{r}=0.63, \mathrm{p}=4 \times 10^{-6}\right)$.

Substantially lower, but in this case significant 2D correlations were obtained between VI and unfamiliar HS (data from Experiment $1 ; \mathrm{r}=0.36, \mathrm{p}=0.001$ ), and between the HS conditions in the two experiments $(r=0.32, \mathrm{p}=0.001)$.

\section{CPGC analyses}

VI-During VI, the CPGC analyses also gave similar results for both experiments (compare top right panels in Figures 1 and 2, and path weights in Tables 9 and 10). They confirmed findings from the GC analysis that the LOCs were driven from prefrontal ROIs, with significant inputs to both LOCs from the OFC in Experiment 1 and both OFC and IFG in 
Experiment 2, further reinforcing the idea of top-down drive into the LOCs. The OFC was an important source of drive in both experiments, additional sources of multiple outputs in Experiment 1 being the IFG, PMd and Pul, while the IFG, PMd and PMv were additional sources of multiple outputs in Experiment 2.

Unfamiliar HS perception-During unfamiliar HS (Figure 1, bottom right panel; Table 11), the CPGC analysis replicated the findings of the GC analysis that the right PCS was the most prominent overall source and the only ROI to drive both LOCs. The right LOC was driven additionally by the left LOC, and the left LOC also received input from avIPS. The PCS regions also drove foci in the IPS, including a path from the right PCS to avIPS. Thus, these findings strengthen the argument for bottom-up connections from somatosensory cortex into the LOCs during HS perception of unfamiliar objects.

Familiar HS perception-Finally, during familiar HS (Figure 2, bottom right panel; Table 12), once again the sources of paths were mainly in frontal cortex, including the OFC, IFG and PMd, as well as a visual cortical area, the MF. The OFC drove the left LOC while the IFG and PMd drove the right LOC; both LOCs received input from the MF. There were additional interactions between these areas, with the MF driving the OFC and the PMd driving the IFG. The LOCs were important as targets in the network. These findings buttress the notion of top-down inputs into the LOCs during HS perception of familiar objects.

Comparisons between tasks-Interestingly, the CPGC analyses eliminated the reciprocal connections seen on the GC analyses. As in the case of the GC analyses, for the CPGC analyses too, the 2D correlation between connectivity matrices was significant for VI and familiar HS ( $r=0.2, p=0.005$, data from Experiment 2) but insignificant between VI and unfamiliar HS $(r=-0.03, p=0.63$, data from Experiment 1$)$ and between the two HS conditions $(r=-0.02, p=0.66)$. Again, this was not simply due to the different subject groups for the two tasks, since the $2 \mathrm{D}$ correlations between the two experiments for the VI task, which also involved different subject groups, was quite high $\left(r=0.76, p=8 \times 10^{-7}\right)$.

\section{Correlations between paths during VI and HS}

We investigated which path weights were significantly positively correlated across subjects between VI and HS. For the data from Experiment 2, comparing VI and familiar HS, in both GC and CPGC analyses, only one path showed a significant correlation - that from the OFC to the left LOC (GC: $r=0.89, p=0.0006$; CPGC: $r=0.96, p=0.0002)$. Similarly, the FC analyses from Experiment 2 revealed a number of paths where the inter-regional correlations were significantly positively correlated between the VI and familiar HS tasks, as well as one negatively correlated path (Table 13).

There were no significant inter-task correlations between VI and unfamiliar HS (data from Expt. 1) for the FC-based inter-regional correlations, for the path weights computed by GC analyses or for the path weights computed by CPGC analyses.

\section{Discussion}

The results of the task-specific connectivity analyses presented here are consistent with our predictions, and converge with the evidence presented in the preceding paper (Lacey et al., 2009) on the conclusion that object VI is strongly linked to HS for familiar, but not for unfamiliar, objects. The underlying neural networks are quite similar during VI and HS perception of familiar objects, with the network underlying HS perception of unfamiliar objects being rather different from either. Further, the EC analyses indicate that hapticallyevoked activation in the LOC during HS perception of familiar objects is instantiated 
through prefrontal inputs as it is for VI, which is consistent with mediation of the LOC activation by VI in this case. For HS perception of unfamiliar objects, in contrast, the EC analyses favor the notion that haptically-evoked activation in the LOC depends on bottomup pathways directly from somatosensory cortex, and thus fit with the idea that a multisensory representation is directly engaged in this case.

All three types of connectivity analysis: FC, GC and CPGC, were essentially consonant. The FC analyses revealed that both VI and familiar HS perception were associated with two segregated networks, one comprising the LOC, the pulvinar and prefrontal cortical areas, and the other involving the PCS and various parts of the IPS. In contrast, the pattern during unfamiliar HS was very different, with numerous interactions and a lack of clearly segregated networks. As noted earlier, such FC-based networks here and in prior studies may include, in addition to true instantaneous neuronal correlations, directional neuronal interactions that are too fast to be reflected in the sluggish BOLD response. Conversely, the GC analyses provide an estimate of directional interactions (EC), but these are potentially contaminated by zero-lag correlations. The CPGC analyses filtered out such zero-lag correlations and offer a conservative estimate of $\mathrm{EC}$, to the extent that the BOLD response is a reflection of the underlying neuronal interactions. In the present study, the CPGC analyses eliminated the reciprocal interactions that were seen on the GC analyses. What is especially interesting is the robustness of the interactions, during both VI and familiar HS, between prefrontal cortical regions and the LOC. These interactions were observed as correlations on the FC analyses and as inputs to the LOC on both the GC and CPGC analyses. In contrast, the PCS, especially on the right, was the primary source of drive to the LOC on both types of EC analysis. These concordant findings indicate that LOC activation during HS perception is rather different depending on whether the palpated objects are familiar or unfamiliar: for familiar objects, LOC activation is mediated by prefrontal cortical drive, in keeping with the top-down generation of a visually imaged object representation. For unfamiliar objects, in contrast, LOC activation is triggered more directly by bottom-up somatosensory input, which accords with the notion of engagement of a modalityindependent object representation. Further, the numerous interactions shown by the FC analysis of HS perception of unfamiliar objects, with especial involvement of parts of the IPS along with the PCS, LOC and prefrontal cortical areas, may imply a role for spatial imagery and shape reconstruction from component parts, as we proposed elsewhere (Lacey et al., 2009).

As we previously suggested, vision and touch probably share a representation of shape that is flexibly accessible via both top-down and bottom-up pathways (Lacey et al., 2007). Its modality-independent engagement during shape perception (Amedi et al., 2001, 2002, 2007; James et al., 2002; Zhang et al., 2004; Stilla and Sathian, 2008) makes the LOC a strong candidate for the locus of this representation. Earlier studies of effective connectivity during HS, using structural equation modeling (Peltier et al., 2007) or multivariate GC analysis (Deshpande et al., 2008), were consistent with this idea. These studies, however, were based on the use of unfamiliar objects only, and also did not analyze EC by task. The multivariate, task-specific FC, GC and CPGC analyses of the present study not only confirm the idea of bidirectional access to the LOC, but also allow us to refine it further, specifying that the connectivity patterns are modulated by object familiarity. This is evidenced by the substantially higher similarity between the connectivity matrices in the VI and familiar HS tasks, compared to that between the connectivity matrices during VI and unfamiliar HS. Further, the familiar HS and VI networks were both characterized by top-down paths from prefrontal foci in the left OFC and IFG into the LOC, without significant bottom-up paths from somatosensory areas into the LOC. Interestingly, many of these paths were reciprocal in the $\mathrm{GC}$ analyses and also present in the $\mathrm{FC}$ analyses, which fits with the idea of ongoing testing of analogies and hypotheses in the OFC (Bar, 2007). Our data are not entirely clear 
as to how the somatosensory inputs arrive in prefrontal areas, but it is probable that they do so indirectly: the GC analysis of familiar HS perception suggests a route left PCS $\rightarrow$ FEF $\rightarrow$ avIPS $\rightarrow$ mSFG and then onto other prefrontal areas. This requires further study. In contrast to the familiar HS network, connectivity to the LOC in the unfamiliar HS network was almost entirely bottom-up from the PCS (Brodmann's area 2 of primary somatosensory cortex [Grefkes et al., 2001]). Thus, depending on the task, modality-independent representations of object shape in the LOC may be accessed either top-down, to support VI, as in the VI and familiar HS tasks, or bottom-up, directly from somatosensory input, as in the unfamiliar HS task.

\section{Conclusions}

VI, and more specifically, visual object imagery, is functionally involved in HS perception of familiar objects: both tasks activate similar networks involving top-down pathways into the LOC from prefrontal areas. By contrast, HS perception of unfamiliar objects activates a very different network involving bottom-up pathways from somatosensory areas into the LOC. These findings from connectivity analyses converge with findings from the preceding paper (Lacey et al., 2009) that VI-evoked activations overlap more, and show stronger correlations, with HS-evoked activation when the felt objects are familiar, compared to unfamiliar. Shape representations in the LOC can therefore be thought of as flexibly accessible by both vision and touch via top-down connections mediating mental imagery, as well as bottom-up paths from early sensory areas. The access route is modulated by object familiarity.

\section{Acknowledgments}

This work was supported by research grants from the NIH (R01 EY12440 and K24 EY17332 to KS, and R01 EB002009 to XH) and NSF (BCS-0519417 to KS). Support to KS from the Veterans Administration is also gratefully acknowledged.

\section{References}

Amedi A, Jacobson G, Hendler T, Malach R, Zohary E. Convergence of visual and tactile shape processing in the human lateral occipital complex. Cereb Cortex. 2002; 12:1202-1212. [PubMed: 12379608]

Amedi A, Malach R, Hendler T, Peled S, Zohary E. Visuo-haptic object-related activation in the ventral visual pathway. Nat Neurosci. 2001; 4:324-330. [PubMed: 11224551]

Amedi A, Stern WM, Camprodon JA, Bermpohl F, Merabet L, et al. Shape conveyed by visual-toauditory sensory substitution activates the lateral occipital complex. Nat Neurosci. 2007; 10:687689. [PubMed: 17515898]

Bar M. The proactive brain: using analogies and associations to generate predictions. Trends Cogn Sci. 2007; 11:280-289. [PubMed: 17548232]

Büchel, C.; Friston, K. Extracting brain connectivity. In: Jezzard, P.; Matthews, PM.; Smith, SM., editors. Functional MRI An Introduction to Methods. Oxford, UK: Oxford University Press; 2001. p. 295-308.

Deshpande G, Hu X, Stilla R, Sathian K. Effective connectivity during haptic perception: A study using Granger causality analysis of functional magnetic resonance imaging data. NeuroImage. 2008; 40:1807-1814. [PubMed: 18329290]

Deshpande G, Sathian K, Hu X. Assessing and Compensating for Zero-lag Correlation Effects in Time-lagged Granger Causality Analysis. 2009 Submitted.

Granger CWJ. Investigating causal relations by econometric models and cross-spectral methods. Econometrica. 1969; 37:424-438.

Grefkes C, Geyer S, Schormann T, Roland P, Zilles K. Human somatosensory area 2: observer independent cytoarchitectonic mapping, interindividual variability, and population map. NeuroImage. 2001; 14:617-631. [PubMed: 11506535] 
James TW, Humphrey GK, Gati JS, Servos P, Menon RS, et al. Haptic study of three-dimensional objects activates extrastriate visual areas. Neuropsychologia. 2002; 40:1706-1714. [PubMed: 11992658]

Lacey S, Campbell C, Sathian K. Vision and touch: Multiple or multisensory representations of objects? Perception. 2007; 36:1513-1521. [PubMed: 18265834]

Lacey S, Flueckiger P, Stilla R, Lava M, Sathian K. Object familiarity modulates involvement of visual imagery in haptic shape perception. 2009 Submitted.

Lacey S, Tal N, Amedi A, Sathian K. A putative model of multisensory object representation. Brain Topog. 200910.1007/s10548-009-0087-4

Mechelli A, Price CJ, Friston KJ, Ishai A. Where bottom-up meets top-down: neuronal interactions during perception and imagery. Cereb Cortex. 2004; 14:1256-1265. [PubMed: 15192010]

Moore T, Armstrong KM. Selective gating of visual signals by microstimulation of frontal cortex. Nature. 2003; 421:370-373. [PubMed: 12540901]

Peltier S, Stilla R, Mariola E, LaConte S, Hu X, et al. Activity and effective connectivity of parietal and occipital cortical regions during haptic shape perception. Neuropsychologia. 2007; 45:476483. [PubMed: 16616940]

Roebroeck A, Formisano E, Goebel R. Mapping directed influence over the brain using Granger causality and fMRI. NeuroImage. 2005; 25:230-242. [PubMed: 15734358]

Stilla R, Deshpande G, LaConte S, Hu X, Sathian K. Posteromedial parietal cortical activity and inputs predict tactile spatial acuity. J Neurosci. 2007; 27:11091-11102. [PubMed: 17928451]

Stilla R, Sathian K. Selective visuo-haptic processing of shape and texture. Hum Brain Mapp. 2008; 29:1123-1138. [PubMed: 17924535]

Stilla R, Hanna R, Hu X, Mariola E, Deshpande G, Sathian K. Neural processing underlying tactile microspatial discrimination in the blind: a functional magnetic resonance imaging study. $\mathrm{J}$ Vis. 2008; 8(10):1-19. 13. 10.1167/8.10.13 [PubMed: 19146355]

Zhang M, Weisser VD, Stilla R, Prather SC, Sathian K. Multisensory cortical processing of object shape and its relation to mental imagery. Cognit Affect Behav Neurosci. 2004; 4:251-259. [PubMed: 15460931] 


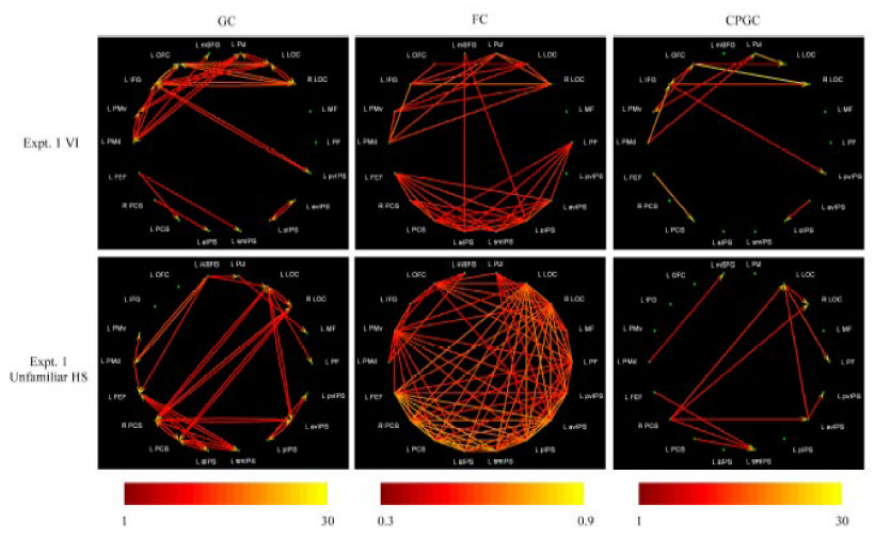

Figure 1.

Significant paths during VI (top row) and unfamiliar HS perception (bottom row), based on data from Experiment 1 of the preceding paper (Lacey et al., 2009). Color scales indicate path weights (arbitrary units) for GC analyses (left column) and CPGC analyses (right column), and correlation coefficients for FC analyses (middle column). 


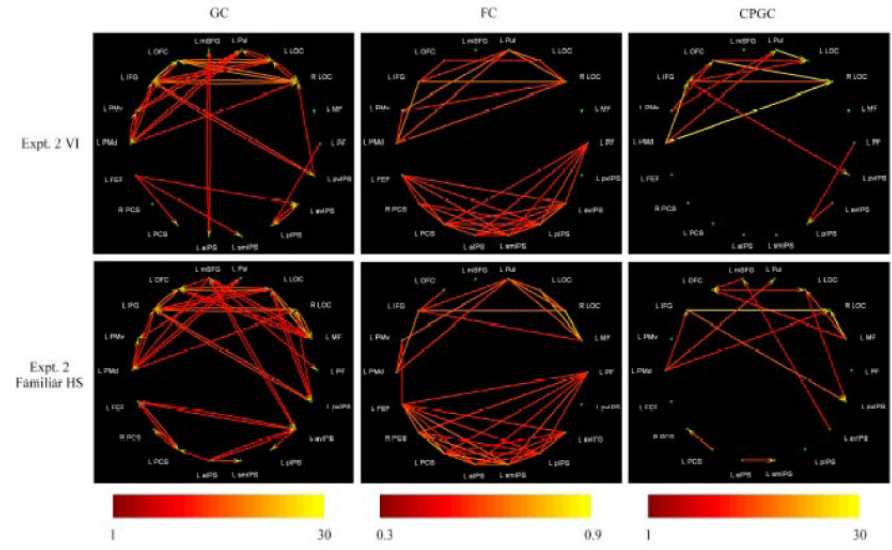

Figure 2.

Significant paths during VI (top row) and familiar HS perception (bottom row), based on data from Experiment 2 of the preceding paper (Lacey et al., 2009). Color scales indicate path weights (arbitrary units) for GC analyses (left column) and CPGC analyses (right column), and correlation coefficients for FC analyses (middle column). 


\begin{tabular}{|c|c|c|c|c|c|c|c|c|c|c|c|c|c|c|c|c|c|c|}
\hline ह & $\stackrel{g}{\stackrel{\theta}{-}}$ & $\begin{array}{l}n \\
0 \\
0\end{array}$ & $\begin{array}{l}+ \\
\stackrel{\infty}{0} \\
\stackrel{0}{0}\end{array}$ & 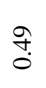 & $\stackrel{\mathbb{2}}{0}$ & $\bar{a}$ & $\stackrel{8}{\circ}$ & $\stackrel{\stackrel{r}{r}}{\dot{m}}$ & $\stackrel{+}{0}$ & $\begin{array}{l}\infty \\
\stackrel{0}{0} \\
0\end{array}$ & ָे & ठे. & $\stackrel{\infty}{n} \stackrel{0}{0}^{2}$ & $\begin{array}{l}\text { O) } \\
0 \\
0\end{array}$ & $\stackrel{\infty}{+}$ & oे & $\stackrel{\infty}{\circ} \stackrel{0}{\circ}$ & \\
\hline 递 & 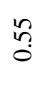 & $\frac{m}{0}$ & $\stackrel{n}{\mathfrak{0}}$ & $\underset{\dot{\sigma}}{\vec{j}}$ & $\frac{6}{0}$ & $\begin{array}{l}\text { ț } \\
\stackrel{0}{0}\end{array}$ & $\hat{o}$ & $\stackrel{?}{\stackrel{0}{0}}$ & $\stackrel{?}{=}$ & $\stackrel{8}{\circ}$ & $\stackrel{5}{0}$ & $\underset{\sigma}{F}$ & 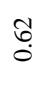 & $\stackrel{?}{0}$ & $\stackrel{\hat{\sigma}}{0}$ & ஸे & & $\stackrel{2}{\hat{0}}$ \\
\hline 告 & 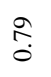 & $\stackrel{n}{\text { In }}$ & f̊ & $\stackrel{t}{\stackrel{0}{0}}$ & $\stackrel{a}{o}$ & $\stackrel{\text { I }}{\circ}$ & $\stackrel{\infty}{\stackrel{\infty}{0}}$ & $\stackrel{\hat{\sigma}}{\circ}$ & $\stackrel{d}{0}$ & $\begin{array}{l}\bar{\infty} \\
\dot{0}\end{array}$ & $\stackrel{\infty}{n}$ & $\begin{array}{l}\infty \\
\stackrel{\infty}{0} \\
\stackrel{0}{0}\end{array}$ & $\stackrel{n}{n}$ & $\overrightarrow{\widetilde{o}}$ & $\stackrel{\overbrace{}}{0}$ & & กิ? & $\stackrel{\infty}{\circ}$ \\
\hline है & $\begin{array}{l}\mathscr{n} \\
\infty \\
0 \\
0\end{array}$ & $\hat{\circ}$ & $\stackrel{\Xi}{\stackrel{d}{0}}$ & $\stackrel{2}{o}$ & $\stackrel{\text { I }}{0}$ & $\hat{0}$ & $\begin{array}{l}\text { Oo } \\
0 \\
0\end{array}$ & ָָ & 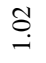 & $\stackrel{t}{0}$ & $\tilde{0}$ & ָ̊ & 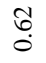 & $\stackrel{n}{2}$ & & $\stackrel{+}{\stackrel{0}{0}}$ & $\stackrel{f}{ \pm}$ & $\stackrel{i}{0}$ \\
\hline 兄 & กี & $\bar{n}$ & f̊. & $\underset{\sigma}{\sigma}$ & $\stackrel{\square}{\circ}$ & $\stackrel{+}{3}$ & $\stackrel{0}{\circ}$ & $\stackrel{\infty}{\stackrel{\infty}{0}}$ & $\stackrel{\Re}{\stackrel{0}{0}}$ & $\exists$ & ర్ర & $\stackrel{\text { ?f }}{\circ}$ & ָ̃ & & $\begin{array}{l}\infty \\
\infty \\
0\end{array}$ & $\stackrel{\text { I }}{0}$ & $\bar{\sigma}_{0}$ & f̊ \\
\hline$\bigcup_{0}^{\infty}$ & $\stackrel{n}{\stackrel{2}{0}}$ & $\stackrel{\infty}{\circ}$ & $\ddot{\circ}$ & $\frac{n}{0}$ & $\hat{o}$ & $\stackrel{g}{\stackrel{f}{0}}$ & $\underset{-}{0}$ & $\begin{array}{l}\mathscr{\infty} \\
0 \\
0\end{array}$ & $\stackrel{\infty}{\stackrel{0}{0}}$ & ले & $\stackrel{2}{\circ}$ & $\stackrel{\infty}{0}$ & & $\stackrel{\infty}{+}$ & $\hat{a}$ & $\tilde{n}$ & $\tilde{\delta}$ & $\tilde{n}$ \\
\hline$\underset{\simeq}{n}$ & $\stackrel{\beth}{\leftrightarrows}$ & $\tilde{0}$ & $\stackrel{\infty}{\stackrel{\infty}{0}}$ & $\overbrace{0}^{\infty}$ & $\underset{0}{J}$ & $\stackrel{J}{0}$ & $\stackrel{0}{0}$ & $\frac{ \pm}{0}$ & $\stackrel{+}{0}$ & $\tilde{n}$ & $\stackrel{\infty}{\circ}$ & & $\stackrel{?}{0}$ & ִִ & ڤ̆ & $\stackrel{\text { }}{0}$ & $\stackrel{m}{\sigma}$ & $\stackrel{8}{\circ}$ \\
\hline 鳬 & $\ddot{n}$ & $\begin{array}{l}\vec{\infty} \\
0\end{array}$ & $\ddot{\sigma}$ & $\vec{m}$ & ָे & $\stackrel{\infty}{\circ}$ & ๙ֶ. & $\overline{\grave{o}}$ & $\stackrel{3}{0}$ & ֶֻ & & $\stackrel{\circ}{\circ}$ & 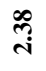 & $\stackrel{\mathbb{2}}{\stackrel{0}{0}}$ & ک్ర & $\stackrel{n}{\circ}$ & $\stackrel{\sim}{\circ}$ & $\frac{9}{0}$ \\
\hline 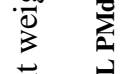 & సै & $\begin{array}{l}\stackrel{8}{0} \\
0\end{array}$ & $\stackrel{5}{\circ}$ & $\stackrel{\text { f }}{\circ}$ & $\stackrel{\text { ?f }}{i}$ & $\stackrel{\hat{b}}{\mathrm{~b}}$ & $\stackrel{\infty}{\stackrel{\infty}{0}}$ & ત્సి & ণั & & $\stackrel{t}{0}$ & $\stackrel{7}{0}$ & $\stackrel{0}{\stackrel{0}{0}}$ & $\stackrel{2}{\stackrel{0}{0}}$ & $\begin{array}{l}\text { Oొ } \\
\stackrel{\infty}{0}\end{array}$ & $\stackrel{+}{n}$ & $\begin{array}{l}\hat{0} \\
0\end{array}$ & $\stackrel{ \pm}{0}$ \\
\hline$\sum_{=}^{=}$ & ֶి & $\stackrel{\leftrightarrow}{\dot{0}}$ & $\stackrel{i}{0}$ & $\stackrel{\text { I }}{\circ}$ & $\underset{i}{\mathbb{i}}$ & $\vec{n}$ & مَّْ & $\stackrel{0}{0}$ & & $\underset{\text { i }}{\text { i }}$ & $\stackrel{\text { fof }}{\circ}$ & $\stackrel{2}{\stackrel{2}{0}}$ & $\overrightarrow{0}$ & $\stackrel{+}{0}$ & $\begin{array}{l}0 \\
\text { : }\end{array}$ & 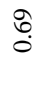 & ô. & $\stackrel{\text { Oे }}{0}$ \\
\hline J & $\stackrel{\substack{\alpha \\
0}}{0}$ & 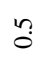 & ลั & $\ddot{r}$ & 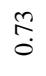 & లి & 9 & & f̊ & 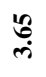 & ?. & $?$ & $\stackrel{n}{\varrho}$ & 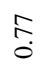 & İ & $\stackrel{a}{o}$ & $\stackrel{\nabla}{0}$ & $\stackrel{\infty}{\stackrel{\sim}{+}}$ \\
\hline $\begin{array}{c}\bar{\Xi} \\
\substack{x \\
\text { In }}\end{array}$ & 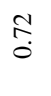 & $\stackrel{\sharp}{\Im}$ & $\stackrel{\infty}{\varrho}$ & ชู & $\stackrel{\overbrace{}}{\stackrel{0}{0}}$ & กี่ & & $\stackrel{\infty}{\stackrel{\infty}{\circ}}$ & $\stackrel{5}{\circ}$ & $\begin{array}{l}\infty \\
0 \\
0\end{array}$ & $\stackrel{.}{\circ}$ & f̊ & ঙे? & ลे & ठै. & $\begin{array}{l}\infty \\
0 \\
0\end{array}$ & $\exists$ & $\stackrel{\simeq}{\leftrightarrows}$ \\
\hline 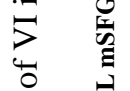 & $\ddot{0}$ & $\underline{\sigma}$ & $\stackrel{\overbrace{}}{\tilde{o}}$ & m़ & $\stackrel{n}{\mathfrak{0}}$ & & $\tilde{n}$ & $\stackrel{5}{0}$ & $\cong$ & $\stackrel{\bullet}{:}$ & $\stackrel{\infty}{+}$ & $\stackrel{\infty}{n}$ & 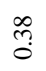 & $\stackrel{a}{\circ}$ & ָ̊ & $\stackrel{l}{:}$ & $\begin{array}{l}\infty \\
\infty \\
0\end{array}$ & $\stackrel{\circ}{\circ}$ \\
\hline 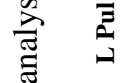 & $\stackrel{t}{\mathbb{0}}$ & $\stackrel{\text { f }}{0}$ & $\stackrel{?}{0}$ & $\vec{i}$ & & $\stackrel{\infty}{\stackrel{\infty}{0}}$ & $\dot{0}$ & $\hat{o}$ & $\begin{array}{l}\bar{b} \\
\text { i }\end{array}$ & $\bar{m}$ & ڤे & $\stackrel{\sim}{0}$ & $\exists$ & $\overrightarrow{3}$ & ָิ & & $\begin{array}{l}\infty \\
\infty \\
0 \\
0\end{array}$ & $\stackrel{n}{\circ}$ \\
\hline لـ & $\stackrel{\imath}{0}$ & 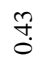 & ָี & & $\stackrel{\tilde{\sigma}}{0}$ & $\stackrel{?}{n}$ & $\stackrel{\Xi}{g}$ & ì & $\stackrel{\Re}{:}$ & ک̊ & $\stackrel{n}{\mathfrak{o}}$ & ले & ?ె? & సे? & $\stackrel{\circ}{\circ}$ & $\begin{array}{l}\text { I } \\
0 \\
0\end{array}$ & $\stackrel{n}{n}$ & $\stackrel{n}{o}$ \\
\hline בְּ & ڤn? & ָิ & & in & $\stackrel{\text { ô }}{0}$ & $\stackrel{尺}{\stackrel{0}{0}}$ & $\stackrel{\text { nh }}{=}$ & $\stackrel{\substack{n\\
}}{ }$ & ñ & $\stackrel{\infty}{0}$ & $\stackrel{\infty}{+}$ & m. & $\overrightarrow{\widetilde{o}}$ & กิ & $\stackrel{ }{\circ}$ & mे & $\stackrel{\sigma}{\sigma}$ & $\frac{5}{0}$ \\
\hline$\sum_{-1}^{5}$ & $\stackrel{\vec{\sigma}}{-}$ & & $\stackrel{+}{0}$ & ک̊ & $\bar{o}_{0}^{\circ}$ & $\stackrel{0}{?}$ & $\stackrel{\text { }}{0}$ & กิ & $\begin{array}{l}\infty \\
\stackrel{0}{0}\end{array}$ & $\vec{m}$ & $\tilde{\tilde{o}}$ & סे & 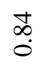 & 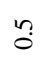 & 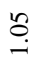 & $\tilde{n}$ & $\stackrel{\overbrace{}}{\text { f }}$ & $\stackrel{8}{\circ}$ \\
\hline 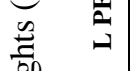 & & $\begin{array}{l}\text { o. } \\
\text { : }\end{array}$ & $\tilde{n}$ & $\stackrel{\sigma}{-}$ & $\begin{array}{l}\stackrel{0}{\infty} \\
0\end{array}$ & กี & $\stackrel{\text { ְै }}{\circ}$ & $\hat{\sigma}$ & ?n? & $\tilde{0}$ & $\begin{array}{l}\infty \\
0 \\
0\end{array}$ & $\stackrel{t}{\mathrm{D}}$ & $\begin{array}{l}\mathscr{\infty} \\
0 \\
0\end{array}$ & $\stackrel{\text { Na }}{0}$ & $\stackrel{0}{0}$ & $\stackrel{\text { }}{\circ}$ & 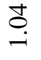 & $\stackrel{0}{\circ}$ \\
\hline$\frac{3}{\bar{E}}$ & 望 & $\sum_{-1}^{n}$ & ્ְత & בِ & $\bar{\Xi}$ & 焉 & ب & لِ & 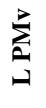 & $\underset{\Xi}{\sum_{a}^{5}}$ & I & $\frac{\tilde{z}}{\approx}$ & $\underbrace{\infty}_{=}$ & בَ & 矛 & 岂 & 㫕 & a \\
\hline
\end{tabular}




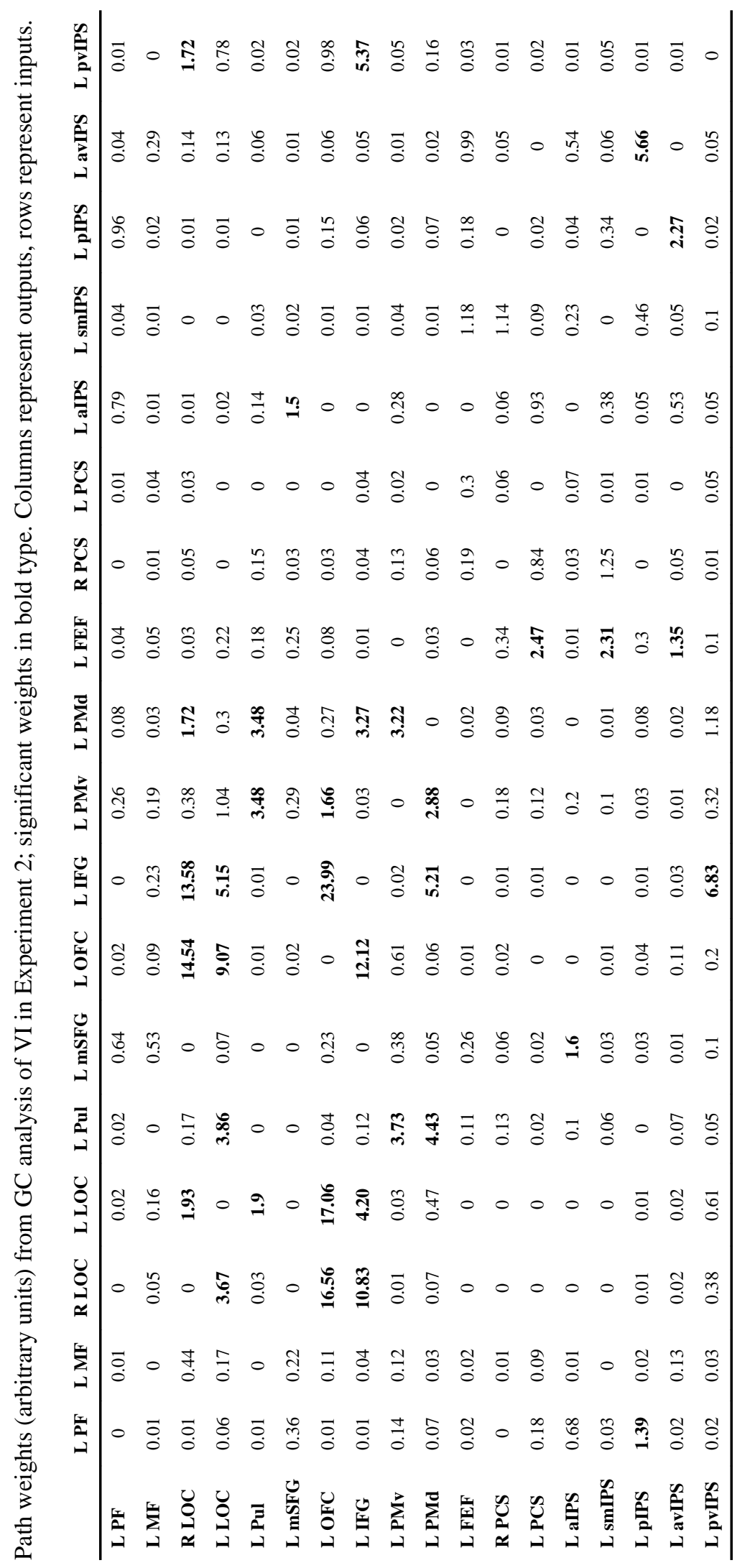




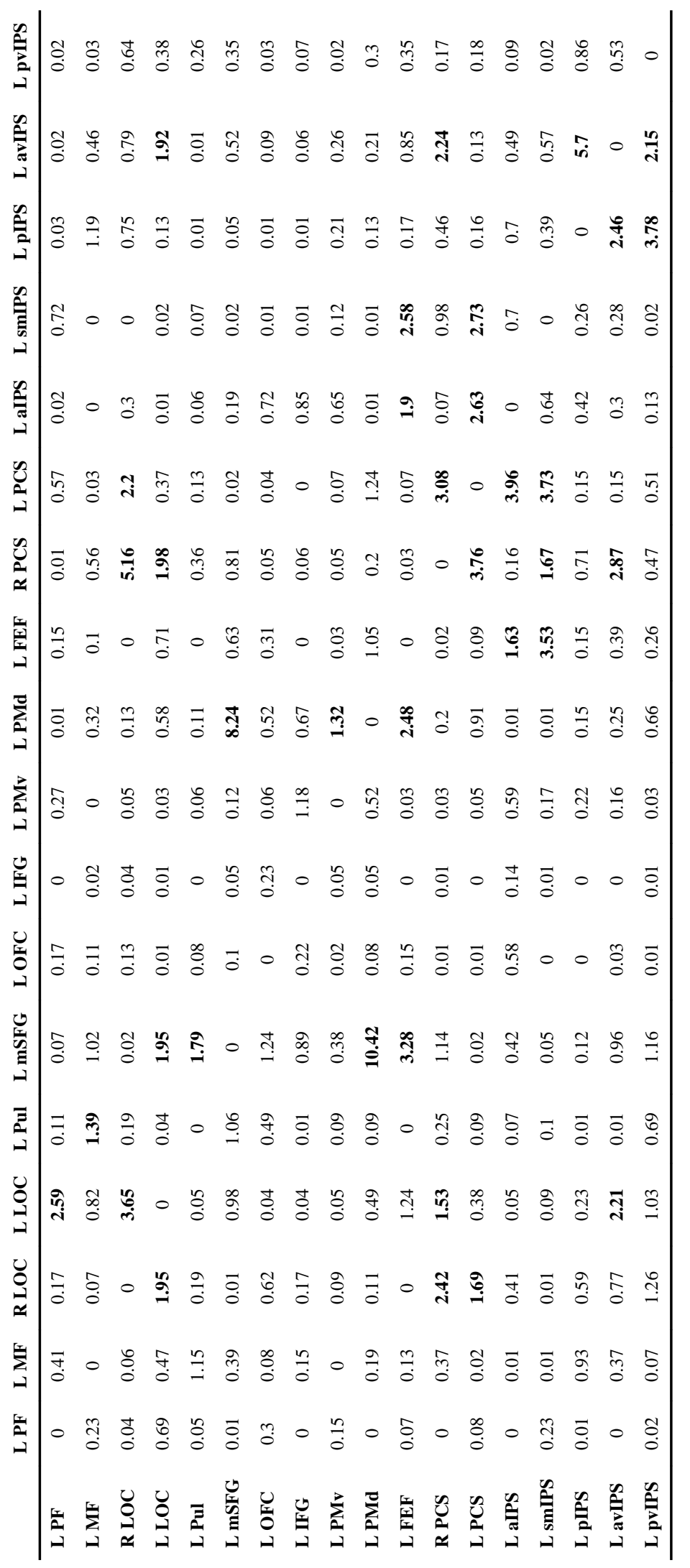




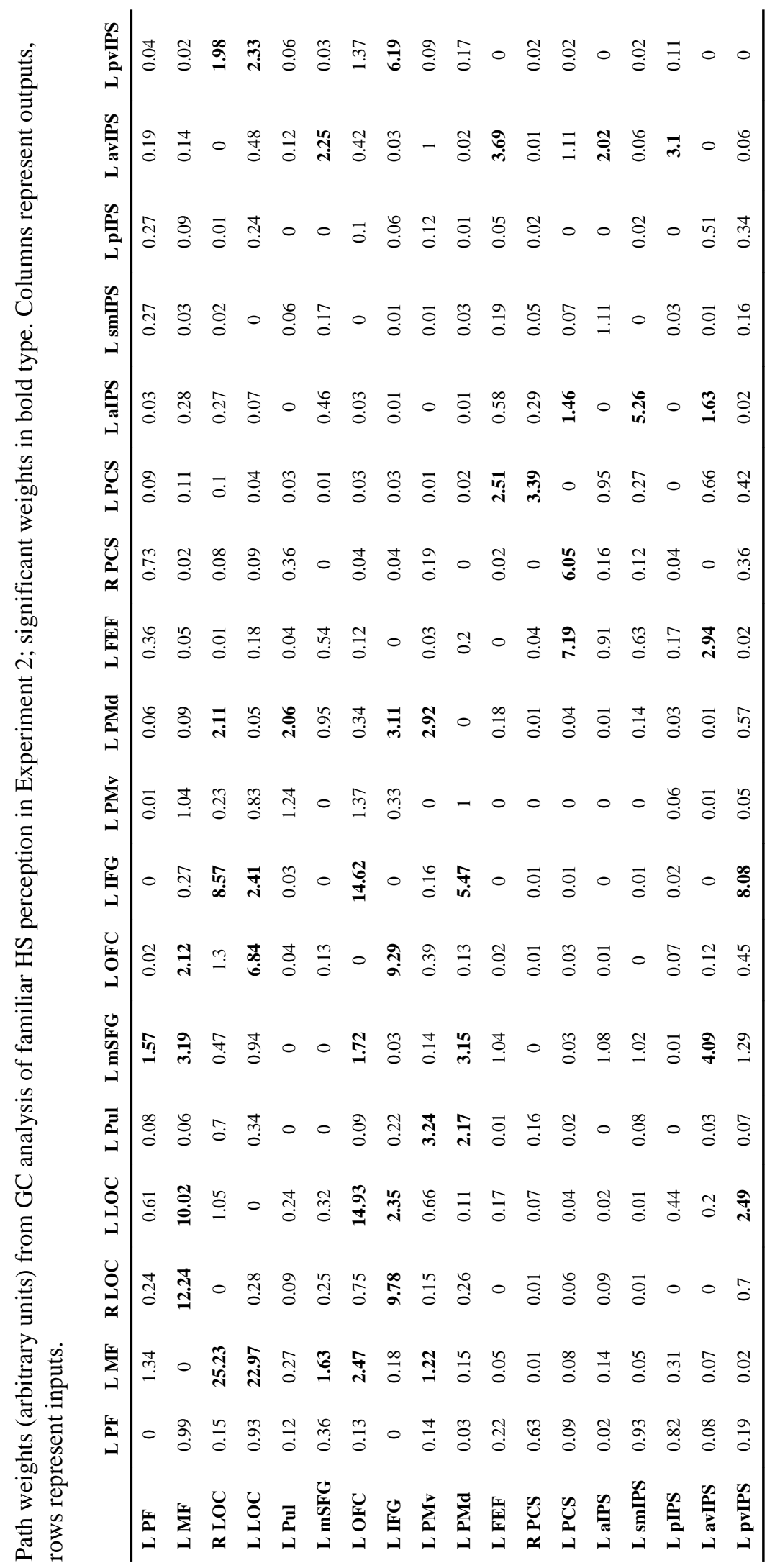




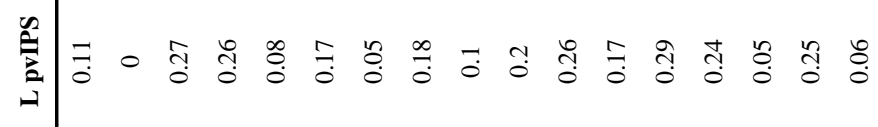

$$
\begin{aligned}
& \text { 言 } \\
& \text { 包 } \\
& \text { 馬 }
\end{aligned}
$$

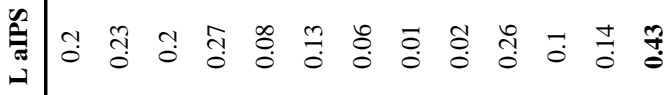

$$
\begin{aligned}
& \text { ஓं }
\end{aligned}
$$

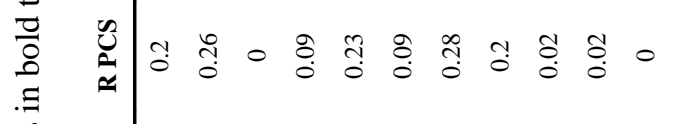




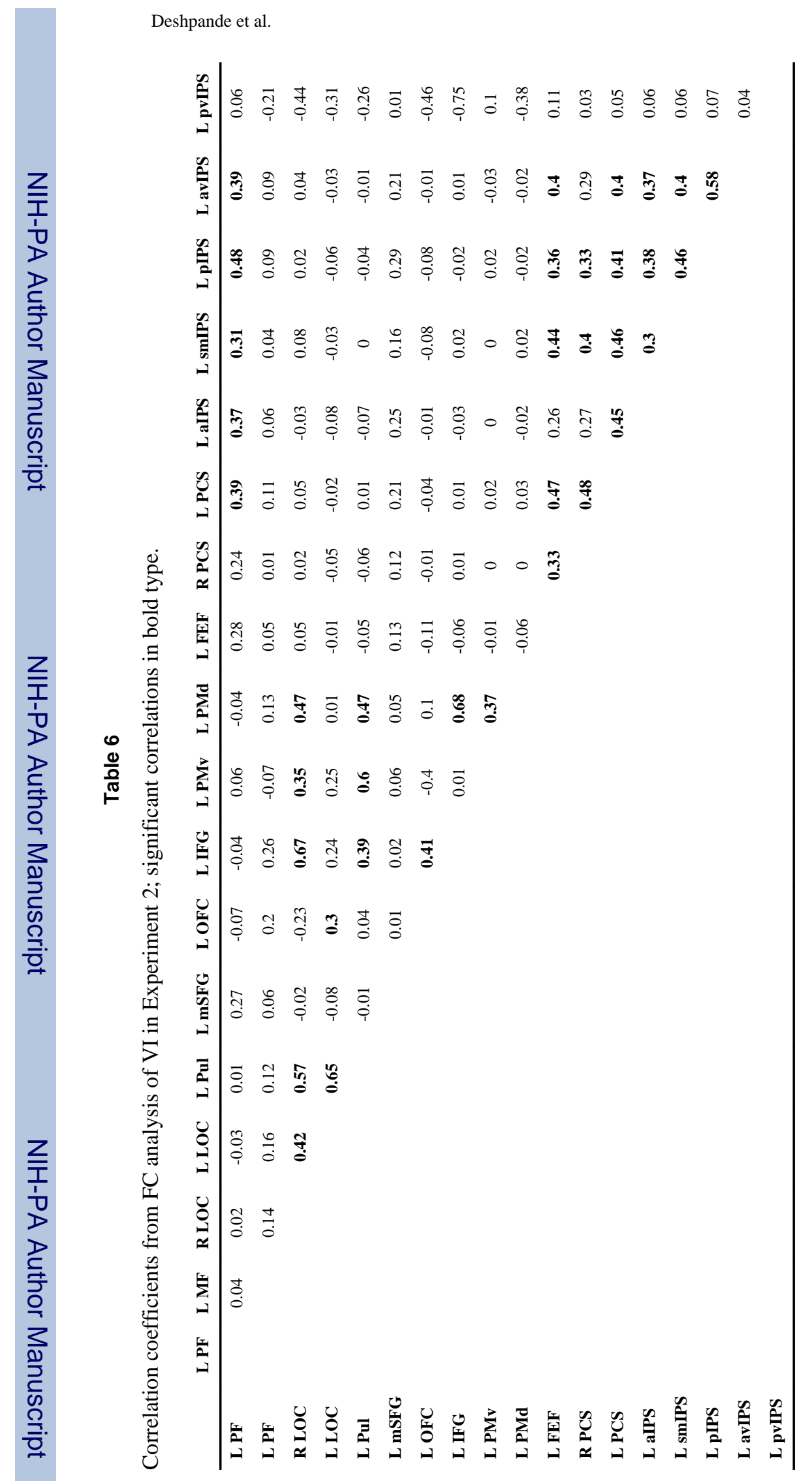

Neuroimage. Author manuscript; available in PMC 2011 April 11. 


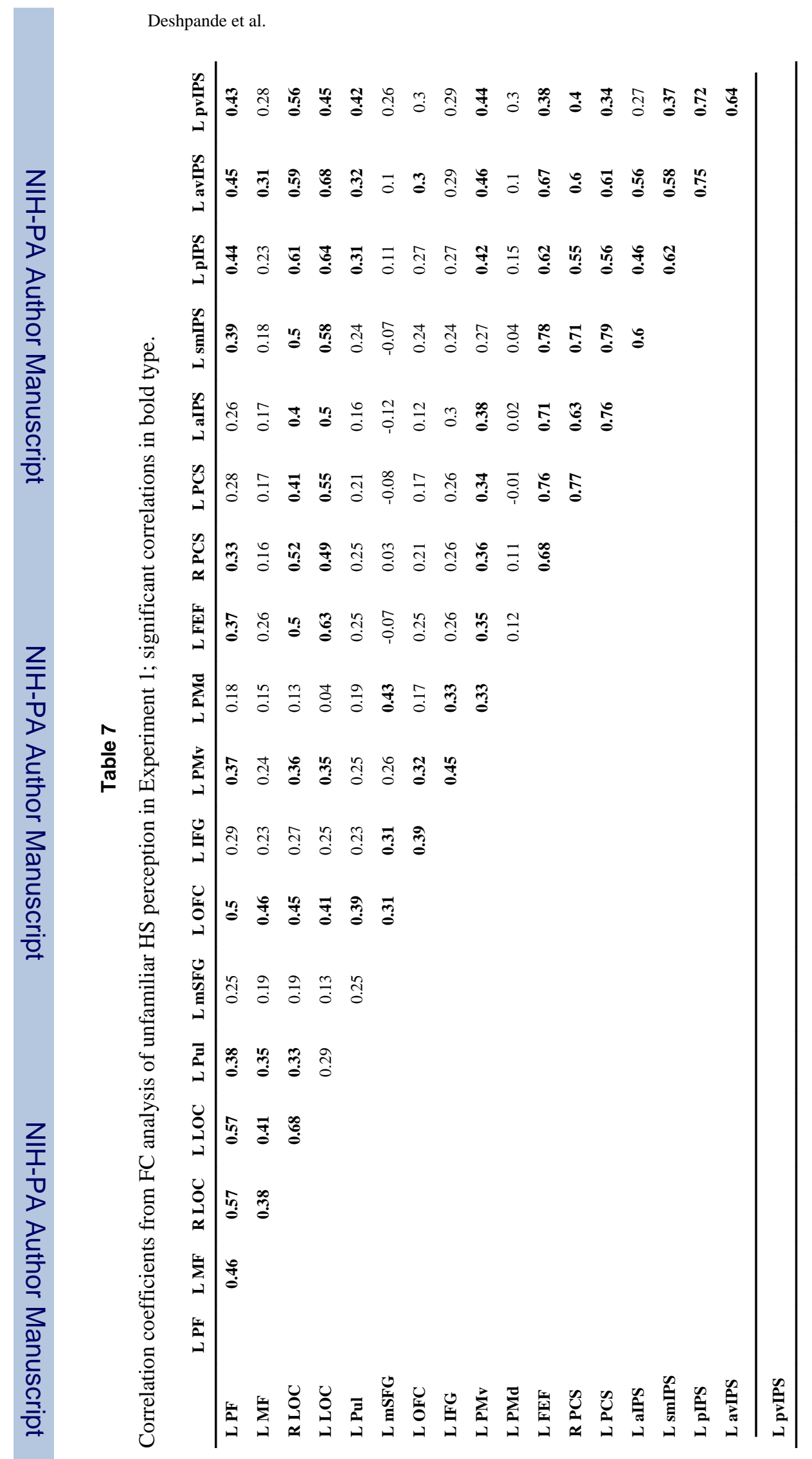

Neuroimage. Author manuscript; available in PMC 2011 April 11. 


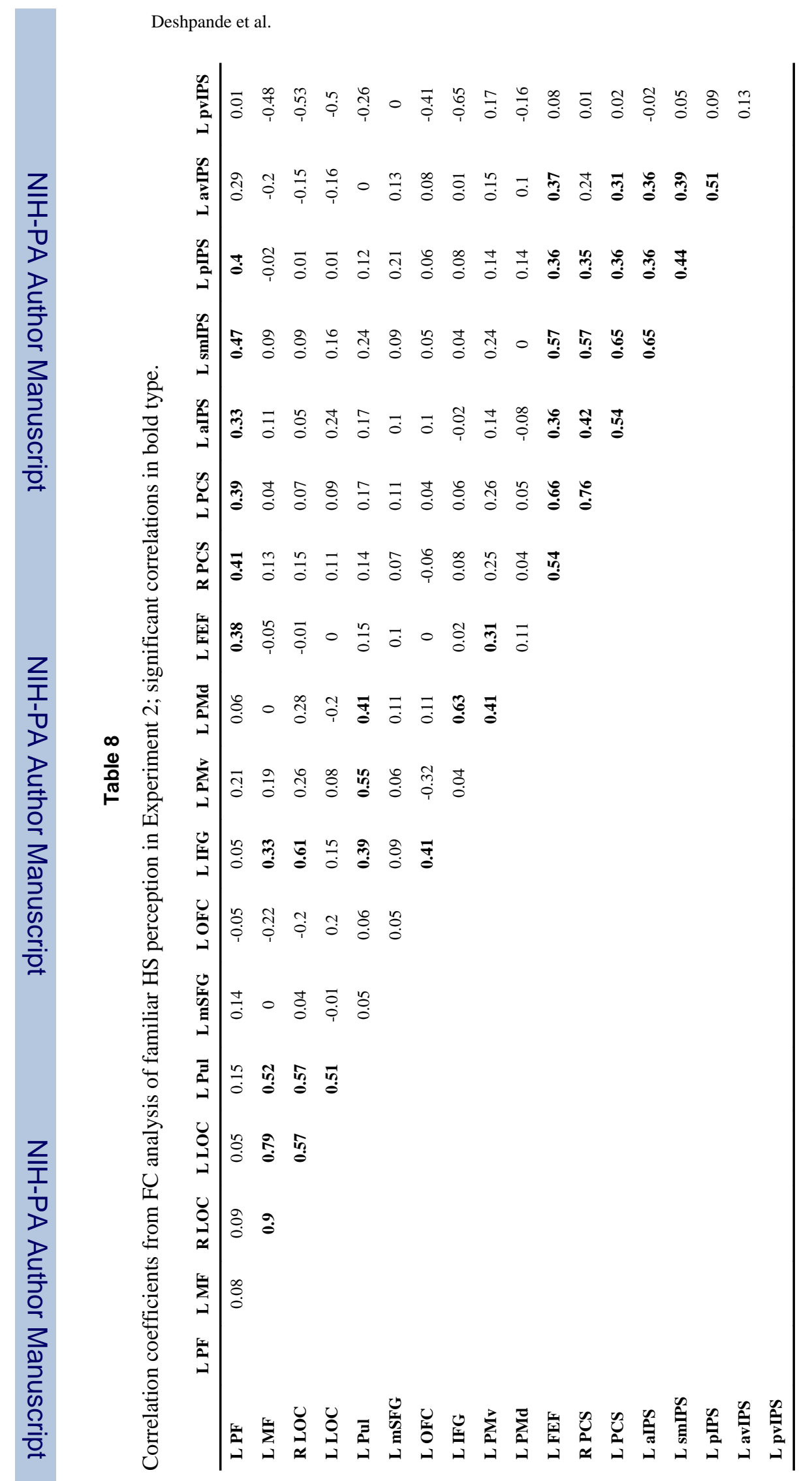

Neuroimage. Author manuscript; available in PMC 2011 April 11. 


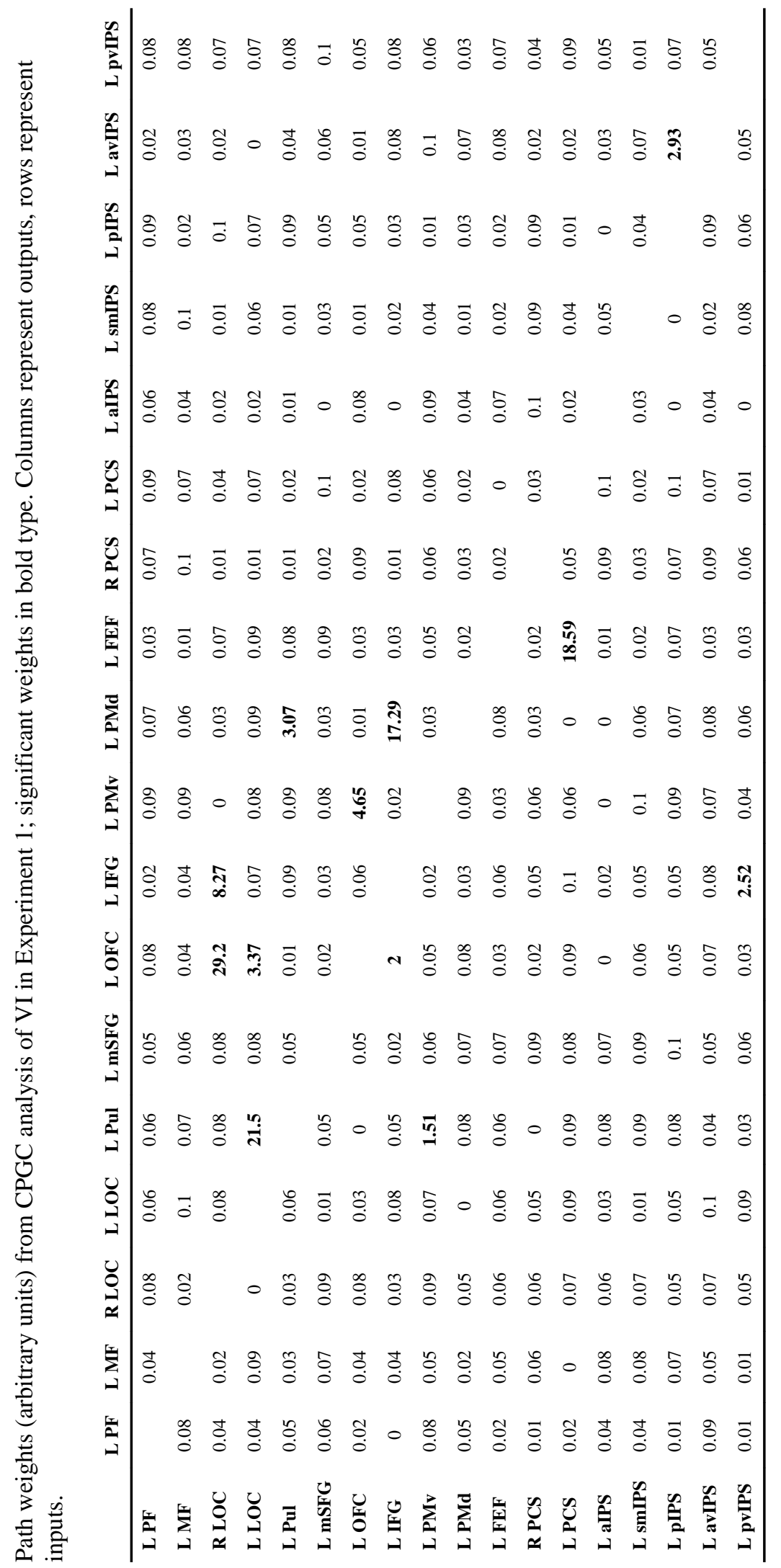




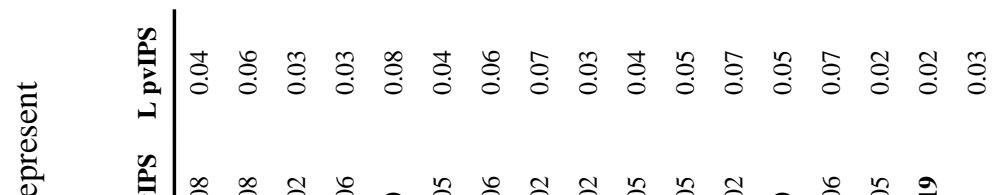

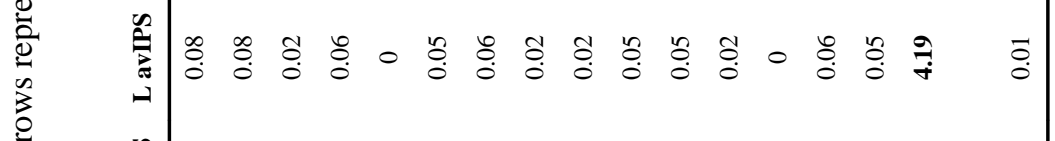

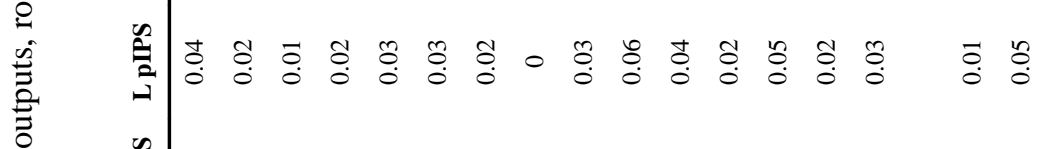

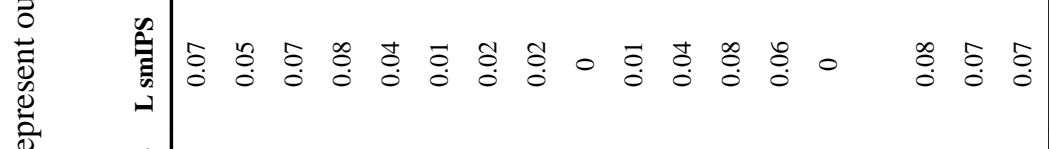

热

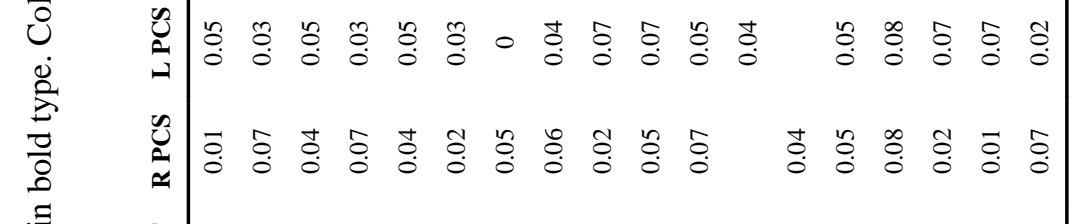

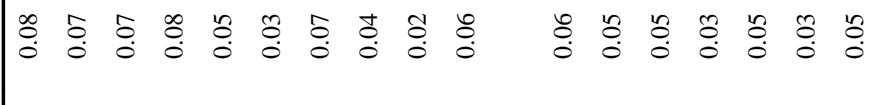

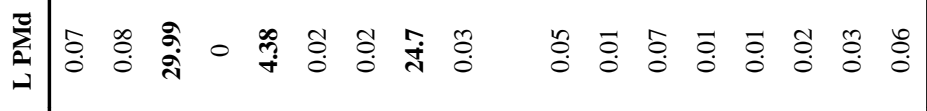

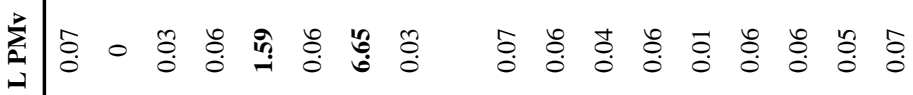

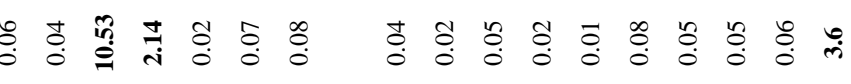

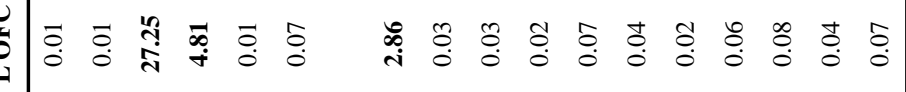

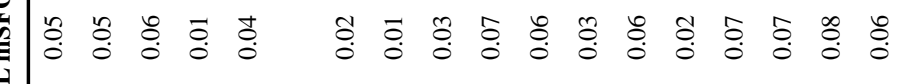

点

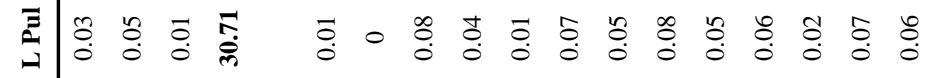

ర্

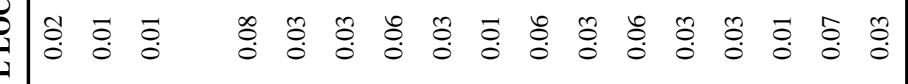

范

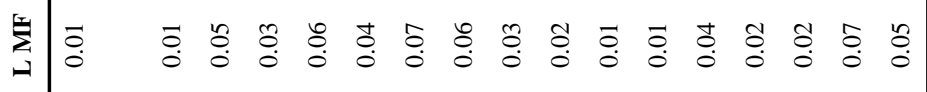

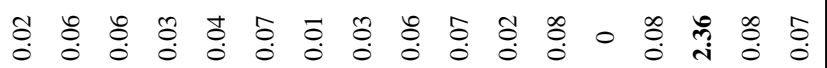

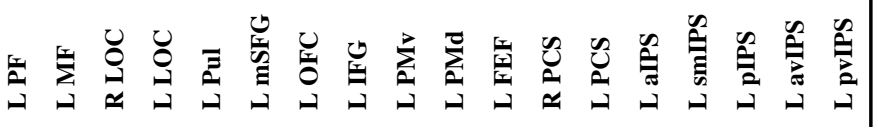




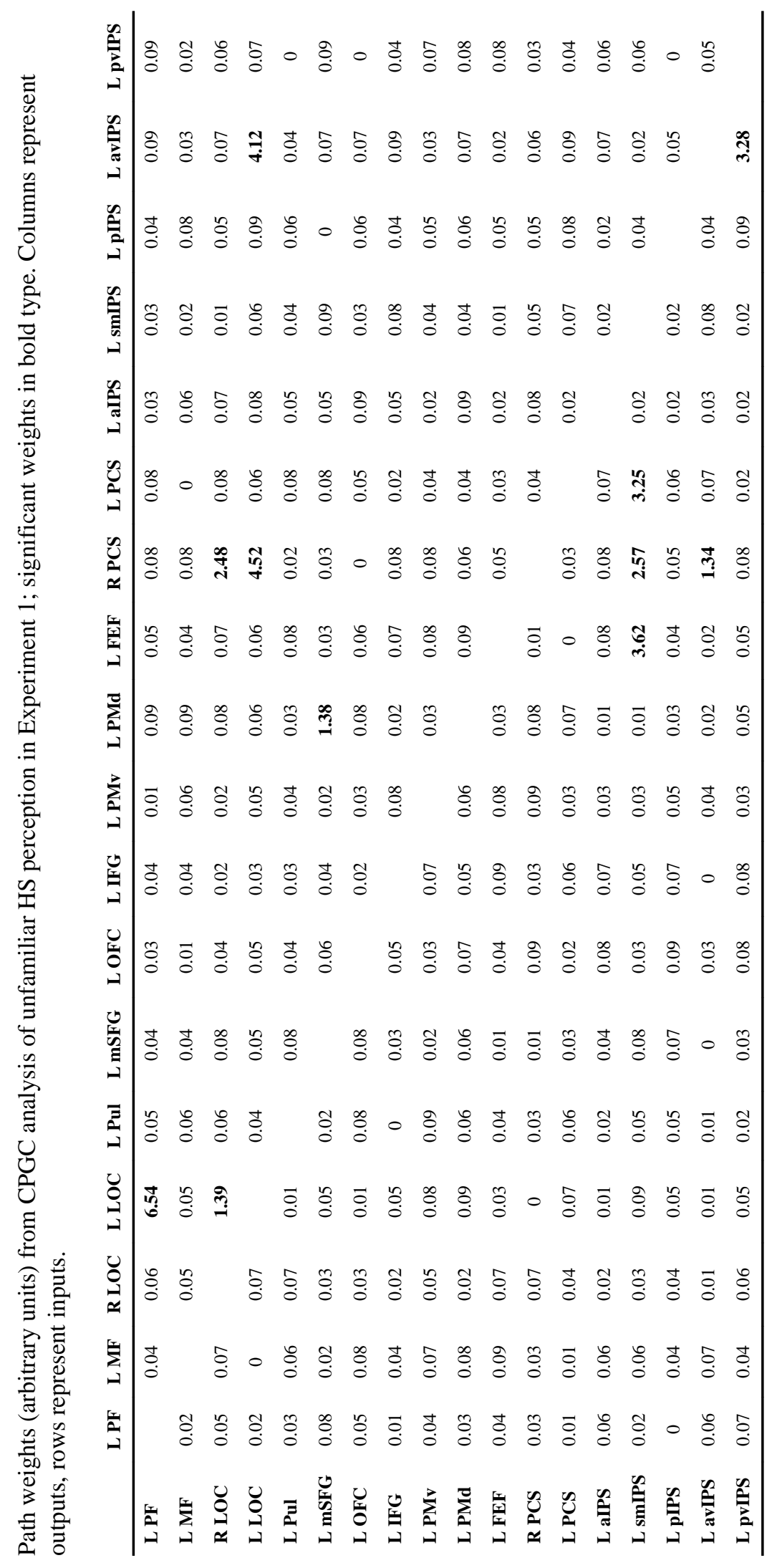




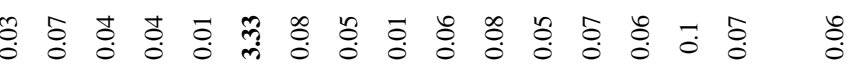
言 茎

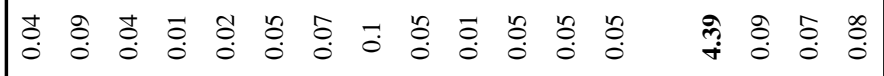
च ป 䱌 吾 띠

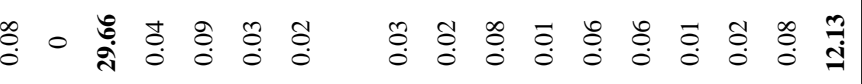

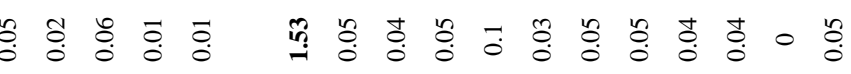

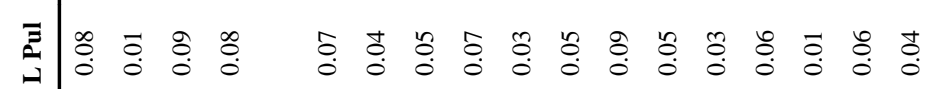
ن

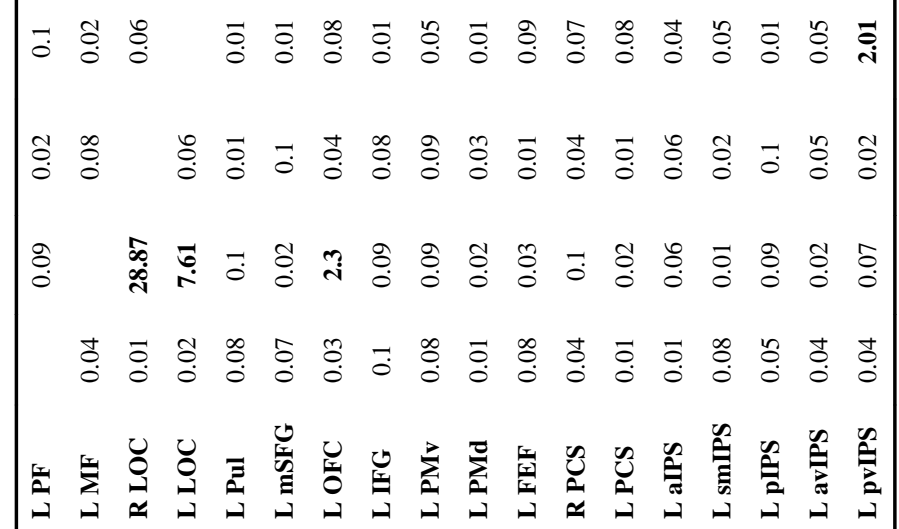




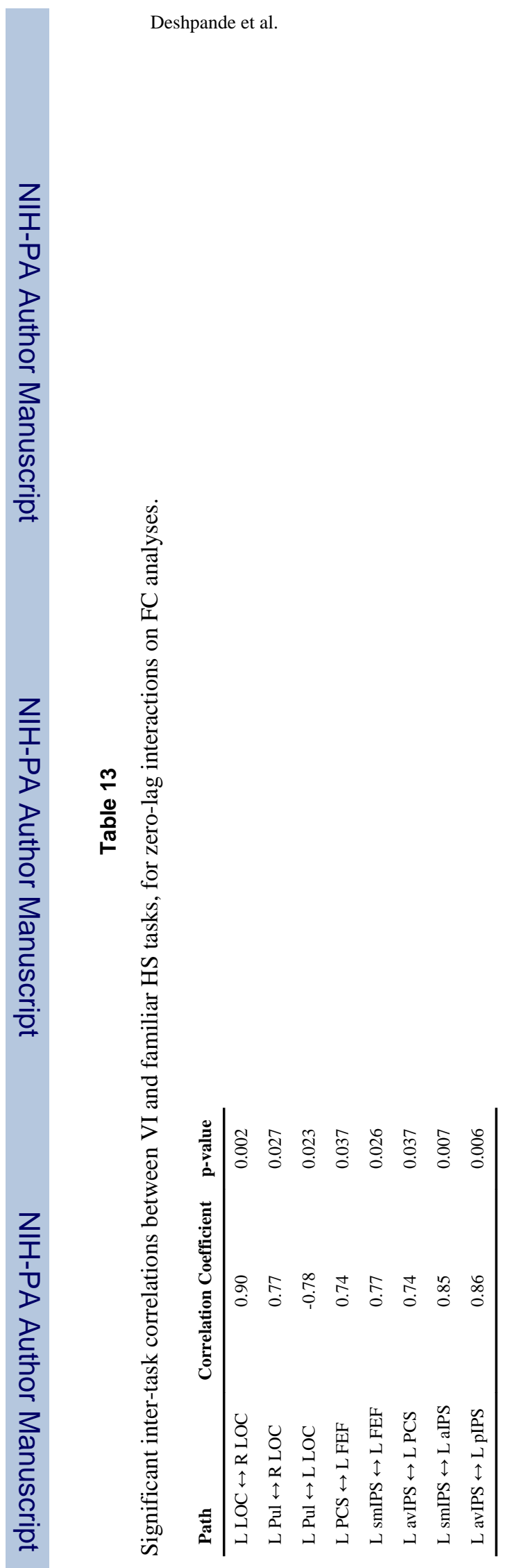

Neuroimage. Author manuscript; available in PMC 2011 April 11. 